\title{
La educación en adultos mayores: percepción de dificultades de aprendizaje y calidad de vida
}

\author{
Autora: Pilar Moreno-Crespo \\ Tipo de tesis: Tesis doctoral \\ Directores: Dr. Luis Amador Muñoz, Dr. Francisco Mateos Claros y Dra.Victoria Pérez de Guzmán Puya \\ Departamento: Departamento de Ciencias Sociales, Facultad de Ciencias Sociales \\ Universidad: Universidad Pablo de Olavide (Sevilla) \\ Programa: Doctorado en Desigualdades e Intervención Social \\ Fecha de presentación: 29 de noviembre de 2011 \\ Recibido: enero 2014
}

\section{RESUMEN}

Nos encontramos en el proceso de metamorfosis de la pirámide poblacional a punta de lanza, que previsiblemente aumentará en altura y supondrá un reto para la sociedad. Estamos presenciando un incrementado del interés en la promoción de la calidad de vida para este sector poblacional, prueba de lo cual son la edición del Libro blanco del envejecimiento activo (Junta de Andalucía, 2010; IMSERso, 2011) y la declaración del año 2012 como Año Europeo del Envejecimiento Activo y la Solidaridad Intergeneracional. Encontramos que atender sus necesidades e inquietudes a nivel educativo toma fuerza y representatividad, ya que se trata de una forma de dar cabida a un colectivo que reclama acceso a la educación. Finalmente, aunque no menos importante, debemos considerar que los adultos mayores han sido estudiados relativamente poco, si tomamos como referencia cualquier otra etapa vital; es decir, debemos tener presente la contribución de esta investigación al desarrollo del conocimiento.

Los objetivos planteados en la investigación son: 1) realizar un estudio sobre el envejecimiento, la calidad de vida y la formación de los adultos mayores y, por otro lado, acercarnos a los programas educativos formales de los adultos mayores (clasificación de Quintana del universo educativo: reglado, formal y sociocultural), y 2) realizar una investigación sobre las dimensiones de aprendizaje y la calidad de vida en este contexto.

El marco teórico da respuesta al primer objetivo planteado analizando, entre otros aspectos, cuestiones terminológicas, sociodemografía, procesos científicos, calidad de vida y educación. Destacamos que uno de los factores más relevantes en el ser humano es el proceso de aprendizaje, como herramienta para la interacción con el medio en el que se desenvuelve, representando un factor fundamental para vivir más tiempo y con mejor calidad de vida.

El marco metodológico responde al segundo objetivo, que se desglosa en los siguientes objetivos específicos: 1) analizar la opinión que poseen los adultos mayores sobre sus dimensiones de aprendizaje; 2) indicar la percepción de los sujetos de estudio sobre la influencia de su participación en un contexto educativo formal en su calidad de vida; 3) descubrir las magnitudes relacionales de las percepciones de los adultos mayores sobre sus dimensiones de aprendizaje y la influencia de la educación formal en 
su calidad de vida, y 4) realizar una propuesta para la mejora de la formación de los adultos mayores. Nuestro posicionamiento paradigmático es positivista y el metodológico es cuantitativo, desarrollando el método de investigación correlacional descriptivo y utilizando como técnica (e instrumento) de recogida de datos la encuesta (el cuestionario).

Destacamos las conclusiones más relevantes en relación con la percepción del adulto mayor en programas educativos formales:

1. No presentan dificultades de atención, memoria, inteligencia, lingüísticas, cálculo o estrategias de aprendizaje; se encuentran motivados y con habilidades sociales suficientes para desenvolverse en el ámbito académico.

2. Los ambientes académico, familiar y social les resultan favorables para el desarrollo de su actividad académico-formal.

3. Reconocen la influencia positiva que ejercen estos en su calidad de vida.

4. Las dimensiones fundamentales que se relacionan entre sí son el nivel formativo (relacionado con el sexo y la ocupación), las dificultades de aprendizaje, la motivación académica, la dimensión personal relacional, el ambiente académico y la calidad de vida.

Las propuestas de actuación quedan planteadas en torno a tres ejes fundamentales:

1. Futuros profesionales: fomento de programas intergeneracionales y formación específica sobre adultos mayores en las titulaciones que profesionalmente tengan vinculación directa o indirecta con estos.

2. Para el diseño de intervenciones socioeducativas: tener presente el sexo y el nivel educativo.

3. Promoción de la educación permanente facilitando el acceso a los diferentes niveles educativos, especialmente a los programas que atiendan sus inquietudes y necesidades, promocionando la participación social.

Consideramos relevante estimular la participación en actividades académicas formales, ya que esto repercute en la promoción de la satisfacción de vida de las personas y, lo que es más relevante, potencia su longevidad con calidad de vida. Los adultos mayores analizados no padecen grandes dificultades de aprendizaje y poseen una alta valoración sobre la percepción del incremento de la calidad de vida gracias a su participación en el programa académico. Concluimos, por lo tanto, que la calidad de vida y el envejecimiento activo se encuentran ligados al concepto de educación permanente, que permite el desarrollo de capacidades superiores en el ser humano, junto con el mantenimiento de las capacidades cognitivas, físicas, sociales, psicológicas y espirituales. La incidencia de todos los aspectos tratados en esta investigación nos aclaran que el adulto mayor sigue en posesión de la capacidad de aprendizaje y que potenciar el mantenimiento y desarrollo de las dimensiones analizadas contribuye al envejecimiento activo.

\section{REFERENCIA BIBLIOGRÁFICA}

Junta de Andalucía (2010). Libro blanco del envejecimiento activo. Sevilla: Junta de Andalucía, Consejería para la Igualdad y el Bienestar Social.

IMSERso (2011). Libro blanco del envejecimiento activo. Madrid: IMSERSo. 